\title{
Effects of short-term cannabidiol treatment on response to social stress in subjects at clinical high risk of developing psychosis
}

\author{
E. Appiah-Kusi ${ }^{1} \cdot$ N. Petros ${ }^{1} \cdot$ R. Wilson ${ }^{1} \cdot$ M. Colizzi ${ }^{1,2} \cdot$ M. G. Bossong ${ }^{1,3} \cdot$ L. Valmaggia $^{4,5} \cdot$ V. Mondelli ${ }^{5,6}$. \\ P. McGuire ${ }^{1,5} \cdot$ S. Bhattacharyya ${ }^{1,5}$ (D)
}

Received: 18 February 2019 / Accepted: 27 December 2019 / Published online: 8 January 2020

(C) The Author(s) 2020

\begin{abstract}
Rationale Stress is a risk factor for psychosis and treatments which mitigate its harmful effects are needed. Cannabidiol (CBD) has antipsychotic and anxiolytic effects.

Objectives We investigated whether CBD would normalise the neuroendocrine and anxiety responses to stress in clinical high risk for psychosis (CHR) patients.

Methods Thirty-two CHR patients and 26 healthy controls (HC) took part in the Trier Social Stress Test (TSST) and their serum cortisol, anxiety and stress associated with public speaking were estimated. Half of the CHR participants were on $600 \mathrm{mg} / \mathrm{day}$ of CBD (CHR-CBD) and half were on placebo (CHR-P) for 1 week.

Results One-way analysis of variance (ANOVA) revealed a significant effect of group (HC, CHR-P, CHR-CBD $(p=.005)$ on cortisol reactivity as well as a significant $(p=.003)$ linear decrease. The change in cortisol associated with experimental stress exposure was greatest in HC controls and least in CHR-P patients, with CHR-CBD patients exhibiting an intermediate response. Planned contrasts revealed that the cortisol reactivity was significantly different in HC compared with CHR-P $(p=.003)$, and in HC compared with CHR-CBD $(p=.014)$, but was not different between CHR-P and CHR-CBD $(p=.70)$. Across the participant groups (CHR-P, CHR-CBD and HC), changes in anxiety and experience of public speaking stress (all $p$ 's $<.02)$ were greatest in the CHR-P and least in the HC, with CHR-CBD participants demonstrating an intermediate level of change.

Conclusions Our findings show that it is worthwhile to design further well powered studies which investigate whether CBD may be used to affect cortisol response in clinical high risk for psychosis patients and any effect this may have on symptoms.
\end{abstract}

Keywords Cannabidiol $\cdot$ Trier Social Stress Test $\cdot$ Ultra-high risk $\cdot$ Psychosis

S. Bhattacharyya

sagnik.2.bhattacharyya@kcl.ac.uk

1 Department of Psychosis Studies, Institute of Psychiatry, Psychology \& Neuroscience (IoPPN), King's College London, PO Box 63, De Crespigny Park, Denmark Hill, London SE5 8AF, UK

2 Department of Neurosciences, Biomedicine and Movement Sciences, Section of Psychiatry, Policlinico “G. B. Rossi”, University of Verona, P.le L.A. Scuro 10, 37134 Verona, Italy

3 Department of Psychiatry, University Medical Centre Utrecht Brain Centre, Utrecht University Utrecht, The Netherlands

4 Department of Psychology, IoPPN, King's College London, London PO Box 77, UK

5 National Institute for Health Research, Biomedical Research Centre, London, UK

6 Department of Psychological Medicine, IoPPN, King's College London, London, UK

\section{Introduction}

Stress plays a major role in the onset and maintenance of psychosis (Pruessner et al. 2017). Exposure to stress in early (Beards et al. 2013) and adult (van Winkel et al. 2008) life has been linked to an increased risk for psychosis. The hypothalamic-pituitary-adrenal (HPA) axis is a key neuroendocrine regulatory system mediating the biological response to stress.

Accumulating evidence suggests that a dysfunction in the HPA-axis might underlie the psychosis continuum (Pruessner et al. 2017). A recent review of the evidence (Appiah-Kusi et al. 2016) suggests that in response to a stressor, patients with established psychosis and those at clinical high risk (CHR) for psychosis (Day et al. 2014; Pruessner et al. 2013) tend to exhibit a blunted cortisol response to both social stress and awakening. Similarly, evidence has shown that 
individuals at CHR of psychosis also have an impaired psychological response to stress. Another study also found that impaired tolerance to stress was more predictive of transition to psychosis than attenuated psychotic symptoms (Yung et al. 2005), showing that the psychological response to stress is an important predictor for psychosis. While atypical antipsychotics have been shown to reduce elevated diurnal cortisol levels (Zhang et al. 2005; Cohrs et al. 2006; Mondelli et al. 2010), antipsychotic treatment has not been shown to correct the blunted cortisol response observed in psychosis (Mondelli et al. 2010).

The harmful effects of cannabis have been mainly attributed to the effects of its main psychoactive ingredient, delta-9tetrahydrocannabinol (THC), which has been shown under experimental conditions to induce psychotic and anxiety symptoms in healthy individuals (D'Souza et al. 2005) and exacerbate psychotic symptoms in patients with preexisting psychosis (D'Souza et al. 2005). In contrast, cannabidiol (CBD), is a safe and well-tolerated (Bergamaschi et al. 2011a) constituent of cannabis and has antipsychotic (Iseger and Bossong 2015; Leweke et al. 2012; McGuire et al. 2017; Bhattacharyya et al. 2010) and anxiolytic (Bergamaschi et al. 2011b) properties. Importantly, preclinical evidence suggests that CBD may attenuate the effects of experimentally induced stress in lab animals following both acute (Guimarães et al. 1990; Campos and Guimarães 2008; Gomes et al. 2011; Gomes et al. 2012) and chronic treatments (Campos et al. 2013). Consistent with this, other data has accumulated that CBD may also block anxiety symptoms induced by THC (Zuardi et al. 1982) or social stress under experimental conditions in healthy volunteers (Zuardi et al. 1993) or patients with social anxiety (Bergamaschi et al. 2011b). Furthermore, CBD has broadly opposite effects to that of THC, both at the behavioural and neural levels (Bhattacharyya et al. 2010; Bhattacharyya et al. 2015; Bhattacharyya et al. 2012) in healthy volunteers and evidence from double-blind randomised clinical trials point towards efficacy as an antipsychotic in patients with established psychosis (Leweke et al. 2012; McGuire et al. 2017). More recently, we have shown that a single dose of CBD may partially normalise dysfunction in the key neural substrates implicated in psychosis in CHR patients (Bhattacharyya et al. 2018). However, whether CBD could attenuate the effects of stress under experimental conditions in patients with CHR has never been tested before. This is of particular importance due to the lack of evidence of a beneficial effect of currently available antipsychotic treatments on the dysregulated neuroendocrine stress response. Therefore, the main objective of the present study was to investigate whether cannabidiol, a non-psychoactive substance present in the extract of the cannabis plant, has potential to mitigate the harmful effects of exposure to stress under experimental conditions in CHR patients.
Therefore, we investigated whether CHR patients had a blunted neuroendocrine and exaggerated anxiety response to acute exposure to social stress compared with healthy controls. Our main aim was to assess whether short-term treatment with CBD would partially normalise the altered acute neuroendocrine and anxiety response stress exposure in CHR patients. We hypothesised that relative to healthy controls, CHR patients under the influence of placebo would display the most severe alterations in the neuroendocrine and psychological responses to stress, while CBD treatment would attenuate some of these effects such that CHR patients under CBD would display an intermediate level of stress response.

\section{Method}

\section{Participants}

Cases consisted of 33 individuals who met the Personal Assessment and Crisis Evaluation (PACE) CHR criteria (Yung et al. 1998), recruited from a specialist clinical service for people at risk for psychosis in South London. Controls consisted of 26 age ( \pm 3 years) and gender-matched healthy individuals, who had no history of a mental disorder, screened negative for psychotic disorder using the Psychosis Screening Questionnaire (PSQ; details below) (Bebbington and Nayani 1995) and were recruited via advertisement in local websites from the same geographical area as cases. All procedures comply with the Helsinki Declaration, as revised in 2008. Participants were reimbursed for their time and travel expenses. These procedures were approved by Psychiatry, Nursing and Midwifery Research Ethics Committee at King's College, London (Approval number PNM/13/14-22), and NHS ethics 13/LO/0243. All participants gave written informed consent before taking part in the study and completed anonymised questionnaires in private.

\section{Procedure}

CHR participants were enrolled in a randomised, placebocontrolled, between-groups, double-blind study (Bhattacharyya et al. 2018). Sixteen participants were given oral $600 \mathrm{mg}$ CBD daily and 16 received identical placebo capsules (STI Pharmaceuticals, UK). On the eighth day of the study, participants took part in the Trier Social Stress Test (TSST). Healthy participants came in for one study session and were not in any drug trial. All participants started the protocol (see Fig. 1) at approximately $10 \mathrm{am}(-60 \mathrm{~min})$ after eating their breakfast at approximately $8.30 \mathrm{am}$. The TSST procedures commenced at approximately $11 \mathrm{am}$. Healthy participants were asked not to use cannabis for $96 \mathrm{~h}$ (4 days) before, alcohol for $24 \mathrm{~h}$ before and nicotine for $6 \mathrm{~h}$ before any other recreational drugs (e.g. speed, cocaine) from 2 weeks 


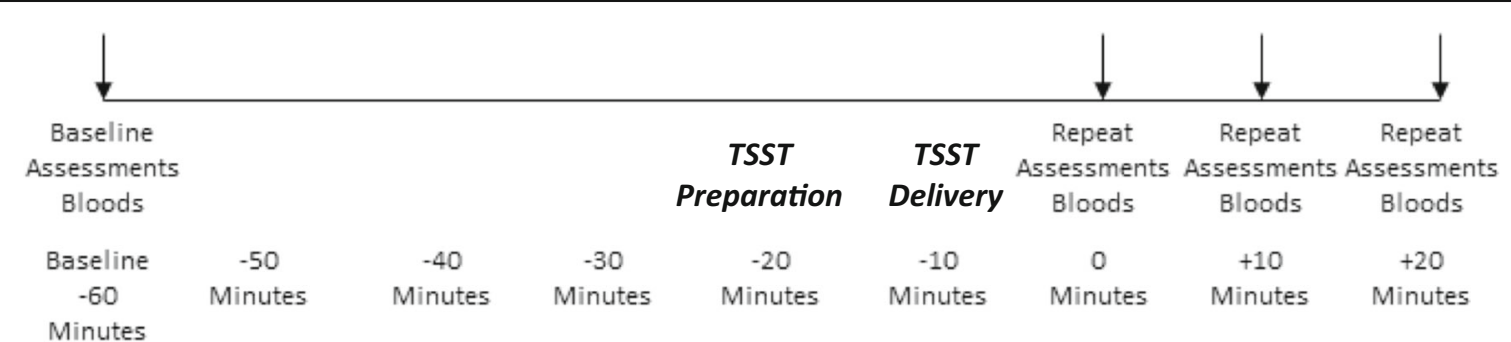

Fig. 1 Timing of procedures for TSST

before the study. CHR participants were asked to abstain from recreational drugs throughout the trial and to not use alcohol for $24 \mathrm{~h}$ before the procedures and nicotine for $6 \mathrm{~h}$ before. All CHR participants were antipsychotic naive. All participants had a clean urine drug screen result. Participants also took part in a short health review to rule out any medical condition which may affect the endocrine system and also to ensure the healthy participants had no underlying mental health condition. Contraceptives were allowed. Participants were seated in a phlebotomy chair and a cannula was then inserted into the antecubital region of the non-dominant arm. Baseline blood samples $(2 \mathrm{ml})$ were collected into serum-separating tubes. The participant then completed the baseline battery of questionnaires (State Trait Anxiety Inventory; STAI, SelfStatements during Public Speaking Scale; SSDPS).

After these had been completed (generally at $11 \mathrm{am} ;-20$ min time point), the participant took part in the TSST (see below). Following this, participants were led back to the phlebotomy chair by the experimenter. Blood samples were obtained immediately and then serial questionnaires (STAI, SSDPS) were filled out $(0 \mathrm{~min})$. This same battery of questionnaires and samples were then also completed at +10 and +20 min after completing the task. At the end of the session, participants were debriefed about the study and received reimbursement for their participation.

\section{Trier Social Stress Test}

The Trier Social Stress Test (TSST) (Kirschbaum et al. 1993) is a well-validated stress induction paradigm that has been shown to reliably induce stress as reflected in changes in cortisol levels, under experimental conditions over the past couple of decades. This stress induction paradigm involves a social evaluative element, which make it comparable to the social stressors which individuals experience in their daily lives and arguably ecologically more valid. Participants were told they will take part in a public speaking exercise. The experimenter takes the participant into a separate room where a panel of two people were assembled and the standardised TSST instructions were read to them (see additional materials; see Fig. 1 for explanation of timing of procedures). They were then taken to a different room for the 10-min preparation period. They were informed that they would be given $10 \mathrm{~min}$ to prepare for a 5-min speech where they had to imagine they had an interview for their ideal job and they needed to deliver the speech to convince the panellists as to why they were ideal for that job, they were then led to an empty room to prepare. After the 10-min preparation period in a different room, participants returned to the panel to deliver their speech. Once they had completed the speech, they were informed they would take part in a mental arithmetic task as per the TSST protocol (see additional materials for details of mental arithmetic task).

\section{Assessment of endocrine response to stress (primary outcome)}

As illustrated in Fig. 1, neuroendocrine response to stress was indexed by measuring serum cortisol level in blood samples collected at baseline $(-60 \mathrm{~min})$ and at $0,+10$ and $+20 \mathrm{~min}$ after the Trier Social Stress Test (TSST).

\section{Assessment of anxiety and negative thoughts about TSST in response to stress}

Anxiety was assessed using the State Trait Anxiety Inventory (STAI) (Spielberger et al. 1970). We employed the 'state' subscale of the questionnaire to measure change in anxiety induced by experimental exposure to stress (STAI-S). Reactions to public speaking were measured using the SelfStatements during Public Speaking Scale (SSDPS) (Hofmann and DiBartolo 2000). This measures individuals' perception of performance in relation to public speaking. It has 5 items evaluating negative thoughts and 5 evaluating positive thoughts, each rated on a 5-point Likert scale. We employed the negative subscale for this study. Both of these scales were collected at baseline $(-60 \mathrm{~min})$ and at $0,+10$ and $+20 \mathrm{~min}$ after the Trier Social Stress Test (TSST).

\section{Statistical analysis}

All statistical analyses were carried out using Statistical Package for Social Science (SPSS) version 22, and the outcome variables were normally distributed. $T$ tests were carried out to assess whether there were group differences in sex or mean age. As there were no differences, neither were 
considered in the analyses. For the outcome variables of anxiety and negative self-statements, area under the curve (AUC) for the four time points was calculated by using the trapezoid formula with respect to ground, as outlined by Pruessner et al. (2003) and these were used in subsequent analyses. For cortisol, the change in cortisol level from baseline to the immediate post-TSST time point (time 0 ; time 0 minus baseline) was used in subsequent analyses. In accordance with our hypothesis, separate one-way analysis of variances (ANOVAs) were carried out with planned linear contrast to examine whether there was a main effect of group (HC, CHR-CBD, CHR-P) such that changes were $\mathrm{HC}>\mathrm{CHR}-\mathrm{CBD}>\mathrm{CHR}-\mathrm{P}$ for each of the main outcomes of interest (cortisol, anxiety and negative self-statements).

\section{Missing data}

Data for one item in the baseline STAI questionnaire was missing in one $\mathrm{HC}$ participant and for one $\mathrm{HC}$ participant in the baseline SSDPS-N. Similarly, data for one item in the SSDPS-N questionnaire for the 0 -min time point was missing in two CHR-CBD participants and one CHR-P, and in one CHR-P participant for the STAI scale at the +10 time point. In the case of missing values in questionnaires, an average of the scale or subscale score was estimated per participant and used in place of the missing value.

In the case of missing data for outcomes that were measured repeatedly, the last observation carried forward method was used to impute missing values. This was used in three instances of missing data in $\mathrm{HC}$ and once in CHR-P for the SSDPS-N and once for CHR-P for cortisol.

Some participants had incomplete data for the different time points and could not realistically be transposed and were therefore not entered into certain analyses. In the cortisol analysis, this occurred in one CHR-CBD participant. For the STAI analysis, this occurred in four healthy controls, five CHR-P participants and two CHR-CBD participants. For the SSDPS$\mathrm{N}$ analysis, this occurred in three healthy controls, five CHR-P participants and two CHR-CBD participants.

\section{Results}

All results are reported as two-tailed tests. One healthy participant was excluded from the analysis as at the time of the baseline assessment, they met criteria for possible psychotic disorder. Table 1 outlines the participants' demographic information as well as baseline psychopathology. This outlines that CHR participants had more cannabis use than healthy controls at baseline but that cannabis use rates were the same between the Placebo and CBD groups. There was also no difference between the two CHR groups on cortisol, STAI and CAARMS positive scores but STAI was significantly higher 
in CHR compared with healthy control participants. Furthermore, there was no significant difference in cortisol levels between the three groups. Healthy controls had a higher level of education than both CHR groups. There was no difference in educational level between CBD and placebo groups. In the CHR-CBD group, on the day of the experiment, mean plasma CBD levels were $61.0 \mathrm{nM}$ (s.d. $=45.49$ ) about an hour before TSST commenced and $74.71 \mathrm{nM}$ (s.d. $=43.65$ ) about 40 min after TSST commenced (which was after TSST was complete).

Table 2 outlines the descriptive statistics for each time point for all of the measures of response to stress.

\section{Cortisol reactions to TSST}

There was a significant effect of group (HC, CHR-P, CHR cannabidiol; $\mathrm{CBD} F(2,54)=5.78, p=.005)$ on cortisol reactivity (measured as the change in the level of cortisol at $0 \mathrm{~min}$ relative to baseline; $-60 \mathrm{~min}$ ). Across the three participant groups, there was a significant $F(1,54)=9.46, p=.003$ linear decrease in cortisol reactivity, such that the change in cortisol (cortisol at time $0 \mathrm{~min}$ relative to baseline; $-60 \mathrm{~min}$ ) associated with experimental stress exposure was greatest in $\mathrm{HC}$ controls and least in CHR-P patients, with CHR-CBD patients exhibiting an intermediate response (Fig. 2). Planned contrasts revealed that the cortisol reactivity was significantly different $(t(54)=3.08, p=.003$; HC v CHR-P mean difference $=$ $117.67,95 \%$ CI $25.45-209.88, d=1.31)$ in $\mathrm{HC}(M=$ $77.08 \mathrm{nmol} / \mathrm{L}$, s.d. $=122.54)$ compared with CHR-P $(M=-$ $40.59 \mathrm{nmol} / \mathrm{L}$, s.d. $=136.98)$, and in HC compared with CHRCBD $(M=-23.67$, s.d. $=99.82 ; t(54)=2.53, p=.014$, mean difference $=100.75,95 \%$ CI $4.94-196.55, d=0.90$ ) but was not different between CHR-P and CHR-CBD $(t(54)=-.39$, $p=.70$, mean difference $=16.92,95 \%$ CI $-86.99-120.84$, $d=0.14)$ ).
Anxiety reactions to acute stress induction with TSST

There was a significant effect of group (HC, CHR-P, CHRCBD $F(2,43)=3.68, p=.034)$ on STAI-S AUC scores. Across the three participant groups, there was a significant $(F(1,43)=6.85, p=.012)$ linear increase in STAI-S scores, indicating that the experience of anxiety in response to the TSST was greatest in CHR-P and least in HC, with CHRCBD exhibiting an intermediate response (Fig. 3). Planned contrasts revealed that the STAI-S AUC was significantly different $(t(43)=-2.62, p=.01 ;$ HC v CHR-P mean difference $=$ $-373.75,95 \% \mathrm{CI}-720.52--26.98, d=0.92)$ in $\mathrm{HC}(M=$ 1428.33 , s.d. $=413.26)$ compared with CHR-P $(M=1802.08$, s.d. $=397.16)$, but not when compared with CHR-CBD $(M=$ 1656.54, s.d. $=359.41, t(43)=-1.64, p=.11 ; \mathrm{HC}$ v CHRCBD mean difference $=-228.21,95 \%$ CI $-566.38-109.97$, $d=0.59)$ and was also not different between CHR-P and CHR-CBD $(t(43)=-.92, p=.36$, mean difference $=-$ 145.54, 95\% CI - 529.16-238.07, $d=0.38$ ) (see Fig. 3).

\section{Effect of acute stress on negative self-statements}

There was a significant effect of group (HC, CHR-P, CHRCBD $F(2,44)=4.57, p=.016)$ on SSDPS-N AUC scores. There was a significant $F(1,44)=9.11, p=.004$ linear increase in SSDPS-N AUC scores indicating that the experience of negative statements was greatest in CHR-P, and least in HC, with CHR-CBD patients exhibiting an intermediate response. Planned contrasts revealed that the SSDPS-N was significantly different $(t(44)=-1.07, p=.004$; HC v CHR-P mean difference $=-169.36,95 \% \mathrm{CI}-309.01--29.71, d=0.94)$ in HC $(M=333.50$, s.d. $=135.82)$ compared with CHR-P $(M=$ 502.86, s.d. $=217.12)$, but not when compared with CHRCBD $(M=394.62$, s.d. $121.58 ; t(44)=-1.12, p=.27$; mean difference $=-61.12,95 \% \mathrm{CI}-203.89-81.66, d=0.47)$ and

Table 2 Descriptives for reactions to stress

\begin{tabular}{|c|c|c|c|c|c|c|c|c|c|c|}
\hline \multirow[b]{2}{*}{ Cortisol levels (nmol/L) } & \multicolumn{2}{|c|}{ Before drug administration } & \multicolumn{2}{|c|}{ Baseline } & \multicolumn{2}{|l|}{$0 \mathrm{~min}$} & \multicolumn{2}{|c|}{$+10 \min$} & \multicolumn{2}{|c|}{$+20 \mathrm{~min}$} \\
\hline & M & s.d. & M & s.d. & M & s.d. & M & s.d. & M & s.d. \\
\hline CHR-CBD & 406.87 & 107.02 & 397.15 & 117.9 & 365.67 & 132.94 & 366.54 & 128.16 & 322.71 & 118.11 \\
\hline CHR-P & 363.82 & 134.02 & 343.47 & 121.02 & 297.47 & 115.07 & 298.73 & 137.77 & 274.27 & 125.76 \\
\hline Healthy control & NA & NA & 369.92 & 162.29 & 447 & 178.34 & 420.72 & 175.83 & 390.8 & 171.31 \\
\hline STAI scores & M & s.d. & M & s.d. & M & s.d. & M & s.d. & M & s.d. \\
\hline CHR-CBD & 39.57 & 9.33 & 37.33 & 8.66 & 44.31 & 11.11 & 42.62 & 10.7 & 43 & 12.44 \\
\hline CHR-P & 41.07 & 8.78 & 38.07 & 10.21 & 48.31 & 11.53 & 44 & 10.82 & 41.13 & 10.76 \\
\hline Healthy control & NA & NA & 32.87 & 10.27 & 40.33 & 13.18 & 34.79 & 11.17 & 31.87 & 10.79 \\
\hline SSDPS-N scores & M & s.d. & M & s.d. & $\mathrm{M}$ & s.d. & M & s.d. & M & s.d. \\
\hline CHR-CBD & NA & NA & 9.27 & 3.94 & 10.77 & 3.59 & 10.54 & 3.8 & 10.5 & 5.36 \\
\hline CHR-P & NA & NA & 10.79 & 4.66 & 13.47 & 5.98 & 13.07 & 6.20 & 11.93 & 5.41 \\
\hline Healthy control & NA & NA & 8 & 2.88 & 9.42 & 4.74 & 8.14 & 3.27 & 7.73 & 3.47 \\
\hline
\end{tabular}


Fig. 2 Group-dependent cortisol reaction

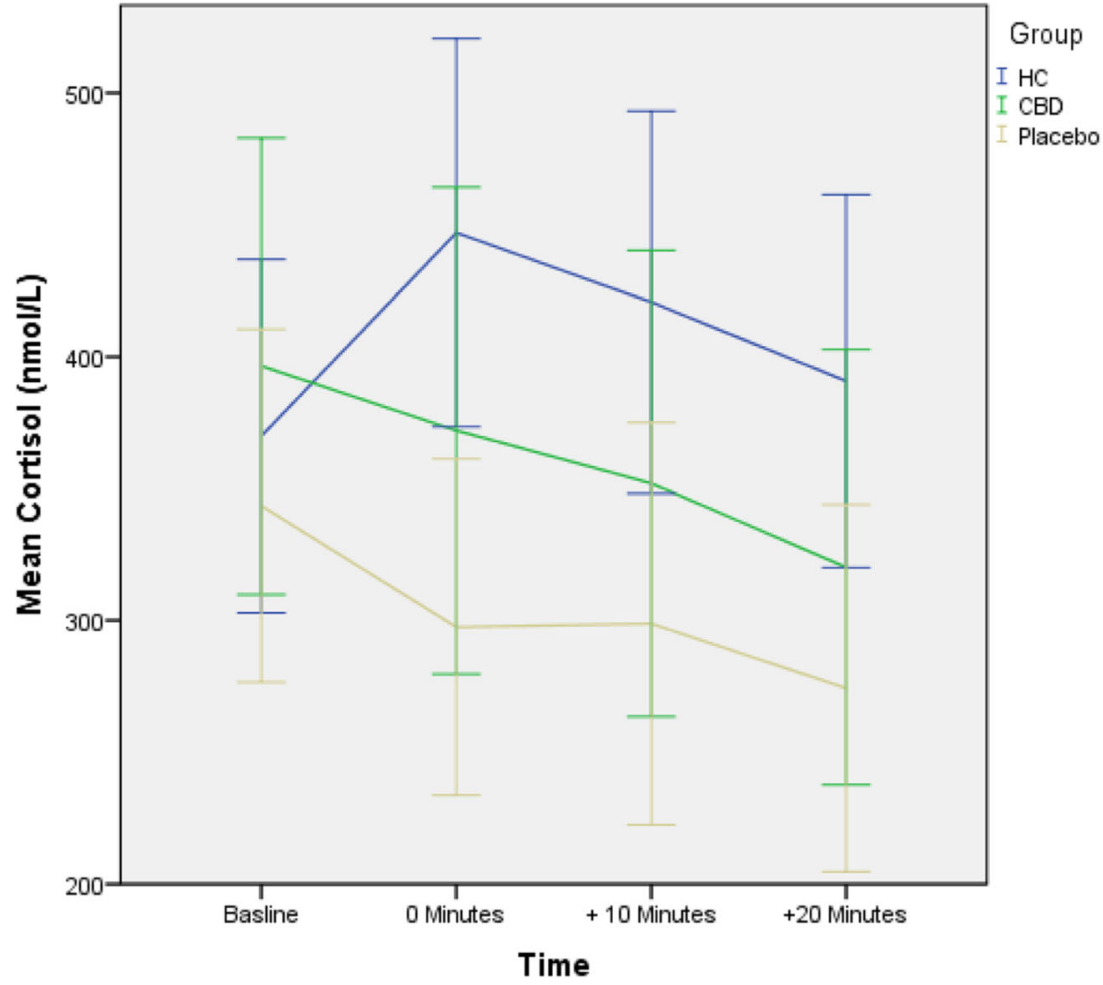

approached a significant difference between CHR-P and CHR-CBD $(t(44)=-1.75, p=.088$ mean difference $=$ $108.24,95 \%$ CI $-46.12-262.60, d=0.62)$.

\section{Discussion}

The first aim of the study was to investigate whether CHR patients responded differently to a social stress paradigm compared with healthy controls. The second aim was to assess

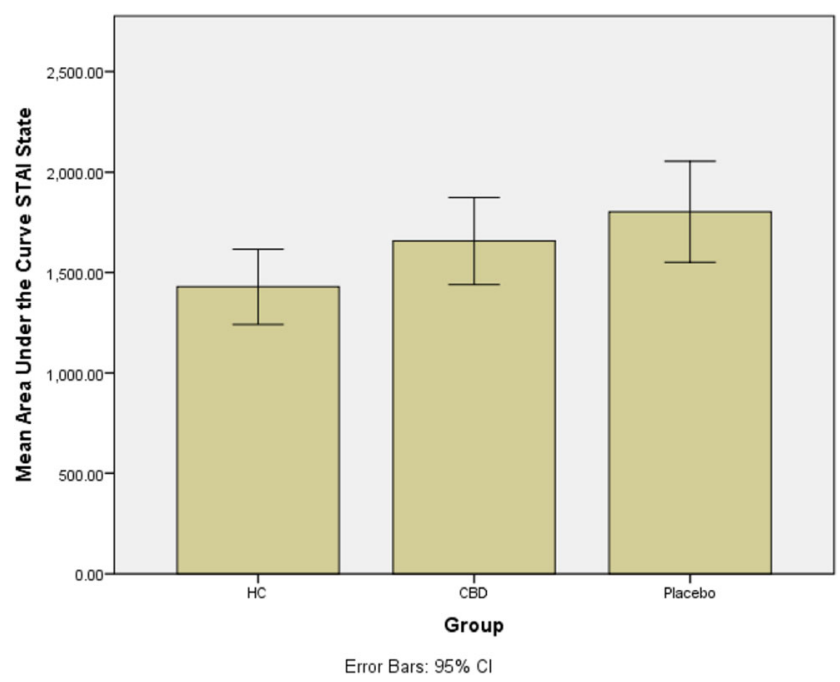

Fig. 3 Group-dependent experience of anxiety whether a 7-day treatment with CBD would attenuate the acute effects of exposure to social stress.

As expected, we found that CHR patients under placebo treatment (CHR-P) had a blunted cortisol response to the Trier Social Stress Test (TSST) compared with healthy controls (HC). Blunted cortisol response to experimental stress in CHR-P compared with HC reported here is consistent with previous reports in CHR individuals (Pruessner et al. 2013) and in those with established psychosis (Jansen et al. 1998). Our report extended these previous results in CHR participants by showing that blunted cortisol response to experimental stress was also associated with a greater psychological response in terms of anxiety and perception of public speaking as stressful in CHR-P compared with HC participants.

In line with our main hypothesis, we also found that CHRCBD displayed intermediate levels of neuroendocrine (cortisol reactivity) and psychological [anxiety and perception of stress (negative self-statements)] response to experimental stress comparedwith CHR-P and HC. Collectively, these findings suggest that CHR participants under placebo displayed abnormal neuroendocrine and psychological responses to experimental stress compared with HC participants, and that 7day treatment with CBD may potentially help partially attenuate these altered responses to experimental stress in CHR participants. However, one needs to be cautious in considering this interpretation, as the significant linear relationship across the 3 participant groups was mainly driven by the significant difference between $\mathrm{HC}$ and CHR-P on these measures. Therefore, further research is needed to investigate whether 
CBD may be used to influence the stress response in early psychosis. Differences in cortisol reactivity and anxiety response to stress in pairwise comparisons between CHR-P and CHR-CBD were not statistically significant, although this difference approached significance when change in perception of stress (negative self-statements) was compared between them. Future longitudinal studies in larger samples taking into account confounders are necessary to independently confirm whether CBD treatment can significantly attenuate altered responses to experimental stress in CHR participants relative to placebo treatment.

Nevertheless, our results provide preliminary evidence that CBD may affect the altered neuroendocrine as well as the psychological responses to acute stress in daily life in CHR patients. Therefore, in contrast to atypical antipsychotics, which have been shown to reduce elevated diurnal cortisol levels (Zhang et al. 2005; Cohrs et al. 2006; Mondelli et al. 2010), but not correct the blunted cortisol response observed in psychosis (Mondelli et al. 2010), CBD may potentially attenuate abnormalities in some of the main components of the acute stress response in the CHR state, future research using larger samples would be required to confirm this suggestion. These results are in line with previous research, which suggests that CBD may have anxiolytic (Bergamaschi et al. 2011b) and antipsychotic effects (Bhattacharyya et al. 2018; Leweke et al. 2012; McGuire et al. 2017).

The mechanism underlying the potential anti-stress effect of CBD is currently unclear, with multiple potential mechanisms being posited. Antipsychotic effects of CBD have been linked to its effects on levels of the endogenous cannabinoid anandamide (AEA) potentially by inhibiting its catalytic enzyme fatty acid amide hydrolase (FAAH). Recent preclinical work has also suggested that CBD may block the anxiogenic effects of chronic stress that was associated with a concomitant decrease in the expression of FAAH following CBD treatment (Fogaça et al. 2018). Anxiolytic effects of CBD were blocked by cannabinoid receptor (type 1 and type 2) antagonists but not by the serotonergic 5HT-1A receptor antagonist. However, other preclinical work suggests an effect of CBD on 5-HT ${ }_{1 \mathrm{a}}$ receptors (Fogaça et al. 2016; Bih et al. 2015; Russo et al. 2005) may underlie its anxiolytic effects.

To the best of our knowledge, this is the first study to have investigated the effects of short-term treatment with CBD on experimentally induced stress in the context of psychosis risk. We employed a well-validated stress induction task that has a long history (Kudielka et al. 2007a) of use for experimental stress induction. This experimental stress paradigm employed social evaluative stress, which may arguably be considered more similar to the kind of life-stress that has been linked with psychosis. While the use of a laboratory task may undermine ecological validity compared with other approaches such as experience sampling techniques, it does allow for more stringent control and standardised stress exposure. Furthermore, the task of speaking in public can be said to be life-like in that it is formed as part of a mock job interview, which is a situation that most people who experience it perceive as stressful. Further, in the healthy population sample, we recruited participants such that they were matched to the at-risk participants.

However, these strengths and the results presented herein need to be considered in light of certain caveats. In particular, we were only able to investigate a relatively modest sample size, which may have affected our ability to detect significant difference between CHR-P and CHR-CBD in pairwise comparisons as well as potential generalisability of these results. Previous studies of a similar nature have employed between 10 and 35 participants (Jansen et al. 1998; Jansen et al. 2000; van Venrooij et al. 2012). Related to this, it may be argued that the dose of CBD employed by us also affected our ability to detect an anxiolytic effect of CBD. A recent study found an anxiolytic effect of an acute dose of CBD at $300 \mathrm{mg}$ but not at 100 or $900 \mathrm{mg}$ (Zuardi et al. 2017), suggesting an inverted Ushaped dose response. However, a previous study (Bergamaschi et al. 2011b) also reported an anxiolytic effect during an experimental public speaking task in patients with social phobia, following a dose of $600 \mathrm{mg}$ of CBD, a dose that we have employed.

Furthermore, in this study, we used venous blood sampling, which in itself can be quite stressful. However, in order to mitigate the effects of venepuncture, we used an atraumatic needle and employed an intravenous cannula that enabled us to avoid repeated venepuncture. While we cannot completely rule out the possibility that the stress of venepuncture may have added to the stress of participants, we do not believe that this would have confounded our results as such stress would have acted across all participants and only added to the social evaluative stress induced by the public speaking task, thereby contributing to how stressed participants felt overall. Furthermore, there was a gap of approximately $50 \mathrm{~min}$ between venepuncture and stress exposure, by which time any effects of stress from the venepuncture would have reduced substantially.

It is also important to note several additional factors may also have affected cortisol response to the Trier Social Stress Test (TSST), which were not controlled for in this study, including body mass index (Bose et al. 2009), night-shift work (Kudielka et al. 2007b), menstrual cycle and the use of contraceptives (Kirschbaum et al. 1999). However, gender distributions between the groups were not significantly different and participants were randomly allocated to the two treatment groups, making it likely that these effects would have affected the study groups to a similar extent. The cortisol levels could also have been affected by individual differences in the diurnal decline of the cortisol slope, but as the CBD and placebo group were randomised, it is reasonable to conclude that these differences would be equal between these groups. Furthermore, in a previous study (Collip et al. 2011), it has 
been shown that the diurnal slope did not differ between healthy controls and those at above average genetic risk for psychosis. The procedure we used for the TSST may also have been insufficient to distinguish between anticipatory and reactive cortisol. The baseline sample was taken $60 \mathrm{~min}$ before the onset of the stressor and the first sample after was taken $20 \mathrm{~min}$ after stressor onset. Previous studies have suggested that anticipatory cortisol levels may provide information when considering differences in mental health and protocols have been developed to capture this (Engert et al. 2013). Similarly, we measured cortisol levels up to $20 \mathrm{~min}$ after the stressful task concluded. It is agreed that cortisol levels reach a peak within 20-30 min after a stressor onset and that they return back to baseline levels within 90 min of cessation of the stressor (Fink 2000). The first sample after cessation of the stressor represents $20 \mathrm{~min}$ after the onset of the stressor; therefore, the + 10-min sample represents 30 min after and +20 min represents $40 \mathrm{~min}$ after the onset of the stressor. It is therefore possible that the sampling is picking up anticipatory cortisol reactions and not reactive cortisol. However, the 0 -min cortisol samples immediately after the TSST represents $20 \mathrm{~min}$ after onset of the stressor and cortisol levels in the healthy control group peak at this point and then continue to reduce, it is possible that this sample is still picking up reactive cortisol. It should also be noted that it has been found that high schizotypes have been found to have a delayed cortisol response (Walter et al. 2018). However, in this study, the peak of the cortisol response was at $15 \mathrm{~min}$ after the cessation of the TSST in both high and low schizotypes, and the delay was in the anticipatory rise. High schizotypes had no rise until $15 \mathrm{~min}$ after the cessation of the TSST whereas the low schizotypes had a rise immediately before the TSST commenced. While it is possible due to the sampling procedure, we may have missed the cortisol peak for the CHR participants, this is unlikely given the last sample is taken a full 40 min after the stressor commenced and no peak is seen in our data. Future research should follow the protocol outlined by Engert et al. (2013) and ensure adequate sampling is taken both in anticipation of the TSST and after the TSST to fully assess the increase and decrease of cortisol in response to stress. In any case, the conclusions of this study that CBD may have a potential to affect cortisol in response to stress is still a possibility.

It could also be argued that education level may affect how stressful the participants found the task, while education level was lower in CHR compared with HC, it was not different between the CBD and placebo groups, making it unlikely that our conclusions would be affect by this difference.

Notwithstanding its limitations, the present study provides a strong rationale for future studies to investigate whether CBD may have potential to mitigate the harmful effects of stress in the course of daily life by attenuating the altered neuroendocrine and psychological responses to acute stress in CHR participants.

Acknowledgements This study represents independent research supported by the National Institute for Health Research (NIHR)/Wellcome Trust King's Clinical Research Facility and the NIHR Biomedical Research Centre and Dementia Unit at South London and Maudsley NHS Foundation Trust and King's College London.

We would like to thank all the participants for taking part in this study, as well as the students and staff past and present for their help in the collection and entering of data, particularly Ewa Klamerus, Enrico Foglia, Eimear Leyden, Sita Parmar, Liam Embliss, Rupa Ramesh, Anand Beri, Maria Calem, Irene Wuersch, Cordelia Watson, Efisia Sais, Noah Yogo and Tabea Schoeler.

Funding Information Dr. Appiah-Kusi was supported by the National Institute for Health Research (NIHR) Collaboration for Leadership in Applied Health Research and Care South London at King's College Hospital NHS Foundation Trust when this work was carried out.

Dr. Bhattacharyya was supported by the National Institute for Health Research (NIHR) through a NIHR Clinician Scientist Award (NIHR CS11-001) when this work was carried out and funding from the Medical Research Council (MRC) (MR/J012149/1) supported this work.

Dr. Matthijs Bossong was supported by a Veni fellowship from the Netherlands Organisation for Scientific Research.

\section{Compliance with ethical standards}

These procedures were approved by Psychiatry, Nursing and Midwifery Research Ethics Committee at King's College, London (Approval number PNM/13/14-22), and NHS ethics 13/LO/0243. All participants gave written informed consent before taking part in the study and completed anonymised questionnaires in private

Conflict of interest The authors declare that they have no conflict of interest.

Disclaimer The views expressed are those of the author(s) and not necessarily those of the NHS, the NIHR or the Department of Health.

Open Access This article is licensed under a Creative Commons Attribution 4.0 International License, which permits use, sharing, adaptation, distribution and reproduction in any medium or format, as long as you give appropriate credit to the original author(s) and the source, provide a link to the Creative Commons licence, and indicate if changes were made. The images or other third party material in this article are included in the article's Creative Commons licence, unless indicated otherwise in a credit line to the material. If material is not included in the article's Creative Commons licence and your intended use is not permitted by statutory regulation or exceeds the permitted use, you will need to obtain permission directly from the copyright holder. To view a copy of this licence, visit http://creativecommons.org/licenses/by/4.0/.

\section{References}

Appiah-Kusi E, Leyden E, Parmar S, Mondelli V, McGuire P, Bhattacharyya S (2016) Abnormalities in neuroendocrine stress response in psychosis: the role of endocannabinoids. Psychol Med 46: $27-45$

Beards S, Gayer-Anderson C, Borges S, Dewey ME, Fisher HL, Morgan C (2013) Life events and psychosis: a review and meta-analysis. Schizophr Bull 39:740-747 
Bebbington P, Nayani T (1995) The psychosis screening questionnaire. Methods in Psychiatric Research, International Journal of

Bergamaschi MM, Queiroz RHC, Chagas MHN, De Oliveira DCG, De Martinis BS, Kapczinski F, Quevedo J, Roesler R, Schröder N, Nardi AE (2011b) Cannabidiol reduces the anxiety induced by simulated public speaking in treatment-naive social phobia patients. Neuropsychopharmacology 36:1219-1226

Bergamaschi M, Mateus, Queiroz RHC, Zuardi AW, Crippa AS (2011a) Safety and side effects of cannabidiol, a Cannabis sativa constituent. Curr Drug Saf 6:237-249

Bhattacharyya S, Morrison PD, Fusar-Poli P, Martin-Santos R, Borgwardt S, Winton-Brown T, Nosarti C, MO'Carroll C, Seal M, Allen P (2010) Opposite effects of $\Delta$-9-tetrahydrocannabinol and cannabidiol on human brain function and psychopathology. Neuropsychopharmacology 35:764

Bhattacharyya S, Crippa JA, Allen P, Martin-Santos R, Borgwardt S, Fusar-Poli P, Rubia K, Kambeitz J, O'carroll C, Seal ML (2012) Induction of psychosis by $\delta 9$-tetrahydrocannabinol reflects modulation of prefrontal and striatal function during attentional salience processing. Arch Gen Psychiatry 69:27-36

Bhattacharyya S, Falkenberg I, Martin-Santos R, Atakan Z, Crippa JA, Giampietro V, Brammer M, McGuire P (2015) Cannabinoid modulation of functional connectivity within regions processing attentional salience. Neuropsychopharmacology 40:1343

Bhattacharyya S, Wilson R, Appiah-Kusi E, O’Neill A, Brammer M, Perez J, Murray R, Allen P, Bossong MG, McGuire P (2018) Effect of cannabidiol on medial temporal, midbrain, and striatal dysfunction in people at clinical high risk of psychosis: a randomized clinical trial. JAMA Psychiatry 75:1107-1117

Bih CI, Chen T, Nunn AVW, Bazelot M, Dallas M, Whalley BJ (2015) Molecular targets of cannabidiol in neurological disorders. Neurotherapeutics 12:699-730

Bose M, Oliván B, Laferrère B (2009) Stress and obesity: the role of the hypothalamic-pituitary-adrenal axis in metabolic disease. Current opinion in endocrinology, diabetes, and obesity 16:340

Campos AC, Guimarães FS (2008) Involvement of 5HT1A receptors in the anxiolytic-like effects of cannabidiol injected into the dorsolateral periaqueductal gray of rats. Psychopharmacology 199:223

Campos AC, de Paula Soares V, Carvalho MC, Ferreira FR, Vicente MA, Brandão ML, Zuardi AW, Zangrossi H, Guimarães FS (2013) Involvement of serotonin-mediated neurotransmission in the dorsal periaqueductal gray matter on cannabidiol chronic effects in paniclike responses in rats. Psychopharmacology 226:13-24

Cohrs S, Röher C, Jordan W, Meier A, Huether G, Wuttke W, Rüther E, Rodenbeck A (2006) The atypical antipsychotics olanzapine and quetiapine, but not haloperidol, reduce ACTH and cortisol secretion in healthy subjects. Psychopharmacology 185:11-18

Collip D, Nicolson NA, Lardinois M, Lataster T, Van Os J, MyinGermeys I (2011) Daily cortisol, stress reactivity and psychotic experiences in individuals at above average genetic risk for psychosis. Psychol Med 41:2305-2315

D'Souza DC, Abi-Saab WM, Madonick S, Forselius-Bielen K, Doersch A, Braley G, Gueorguieva R, Cooper TB, Krystal JH (2005) Delta9-tetrahydrocannabinol effects in schizophrenia: implications for cognition, psychosis, and addiction. Biol Psychiatry 57:594-608

Day FL, Valmaggia LR, Mondelli V, Papadopoulos A, Papadopoulos I, Pariante CM, McGuire P (2014) Blunted cortisol awakening response in people at ultra high risk of developing psychosis. Schizophr Res 158:25-31

Engert V, Efanov SI, Duchesne A, Vogel S, Corbo V, Pruessner JC (2013) Differentiating anticipatory from reactive cortisol responses to psychosocial stress. Psychoneuroendocrinology 38:1328-1337

Fink, George. 2000. Encyclopedia of stress (academic press)

Fogaça, Manoela V, Alline C Campos, and Francisco S Guimarães. 2016. 'Cannabidiol and 5-HT 1A receptors.' in, Neuropathology of Drug Addictions and Substance Misuse (Elsevier)
Fogaça MV, Campos AC, Coelho LD, Duman RS, Guimarães FS (2018) The anxiolytic effects of cannabidiol in chronically stressed mice are mediated by the endocannabinoid system: role of neurogenesis and dendritic remodeling. Neuropharmacology 135:22-33

Gomes FV, Resstel LBM, Guimarães FS (2011) The anxiolytic-like effects of cannabidiol injected into the bed nucleus of the stria terminalis are mediated by 5-HT1A receptors. Psychopharmacology 213:465-473

Gomes FV, Reis DG, Alves FHF, Corrêa FMA, Guimaraes FS, Resstel LBM (2012) Cannabidiol injected into the bed nucleus of the stria terminalis reduces the expression of contextual fear conditioning via 5-HT1A receptors. J Psychopharmacol 26:104-113

Guimarães FS, Chiaretti TM, Graeff FG, Zuardi AW (1990) Antianxiety effect of cannabidiol in the elevated plus-maze. Psychopharmacology 100:558-559

Hofmann SG, DiBartolo PM (2000) An instrument to assess selfstatements during public speaking: scale development and preliminary psychometric properties. Behav Ther 31:499-515

Iseger TA, Bossong MG (2015) A systematic review of the antipsychotic properties of cannabidiol in humans. Schizophr Res 162:153-161

Jansen LMC, Gispen-de Wied CC, Gademan PJ, De Jonge RCJ, van der Linden JA, Kahn RS (1998) Blunted cortisol response to a psychosocial stressor in schizophrenia. Schizophr Res 33:87-94

Jansen LMC, Gispen-de Wied CC, Kahn RS (2000) Selective impairments in the stress response in schizophrenic patients. Psychopharmacology 149:319-325

Kirschbaum C, Pirke K-M, Hellhammer DH (1993) The 'Trier Social Stress Test'-a tool for investigating psychobiological stress responses in a laboratory setting. Neuropsychobiology 28:76-81

Kirschbaum C, Kudielka BM, Gaab J, Schommer NC, Hellhammer DH (1999) Impact of gender, menstrual cycle phase, and oral contraceptives on the activity of the hypothalamus-pituitary-adrenal axis. Psychosom Med 61:154-162

Kudielka BM, Buchtal J, Uhde A, Wüst S (2007b) Circadian cortisol profiles and psychological self-reports in shift workers with and without recent change in the shift rotation system. Biol Psychol 74:92-103

Kudielka BM, Hellhammer DH, Kirschbaum C, Harmon-Jones E, Winkielman P (2007a) Ten years of research with the Trier social stress test-revisited. Social neuroscience: Integrating biological and psychological explanations of social behavior:56-83

Leweke FM, Piomelli D, Pahlisch F, Muhl D, Gerth CW, Hoyer C, Klosterkötter J, Hellmich M, Koethe D (2012) Cannabidiol enhances anandamide signaling and alleviates psychotic symptoms of schizophrenia. Transl Psychiatry 2:e94

McGuire, Philip, Philip Robson, Wieslaw Jerzy Cubala, Daniel Vasile, Paul Dugald Morrison, Rachel Barron, Adam Taylor, and Stephen Wright. 2017. 'Cannabidiol (CBD) as an adjunctive therapy in schizophrenia: a multicenter randomized controlled trial', American Journal of Psychiatry: appi. ajp. 2017.17030325

Mondelli V, Dazzan P, Hepgul N, Di Forti M, Aas M, D’Albenzio A, Di Nicola M, Fisher H, Handley R, Marques TR, Morgan C, Navari S, Taylor H, Papadopoulos A, Aitchison KJ, Murray RM, Pariante CM (2010) Abnormal cortisol levels during the day and cortisol awakening response in first-episode psychosis: the role of stress and of antipsychotic treatment. Schizophr Res 116:234-242

Pruessner JC, Kirschbaum C, Meinlschmid G, Hellhammer DH (2003) Two formulas for computation of the area under the curve represent measures of total hormone concentration versus time-dependent change. Psychoneuroendocrinology 28:916-931

Pruessner M, Béchard-Evans L, Boekestyn L, Iyer SN, Pruessner JC, Malla AK (2013) Attenuated cortisol response to acute psychosocial stress in individuals at ultra-high risk for psychosis. Schizophr Res 146:79-86

Pruessner M, Cullen AE, Aas M, Walker EF (2017) The neural diathesisstress model of schizophrenia revisited: an update on recent findings 
considering illness stage and neurobiological and methodological complexities. Neurosci Biobehav Rev 73:191-218

Russo EB, Burnett A, Hall B, Parker KK (2005) Agonistic properties of cannabidiol at 5-HT1a receptors. Neurochem Res 30:1037-1043

Spielberger, Charles Donald, Richard L Gorsuch, and Robert E Lushene. 1970. 'Manual for the state-trait anxiety inventory'

van Venrooij JAEM, Fluitman SBAHA, Lijmer JG, Kavelaars A, Heijnen CJ, Westenberg HGM, Kahn RS, Gispen-de Wied CC (2012) Impaired neuroendocrine and immune response to acute stress in medication-naive patients with a first episode of psychosis. Schizophr Bull 38:272-279

van Winkel R, Stefanis NC, Myin-Germeys I (2008) Psychosocial stress and psychosis. A review of the neurobiological mechanisms and the evidence for gene-stress interaction. Schizophr Bull 34:1095-1105

Walter EE, Fernandez F, Snelling M, Barkus E (2018) Stress induced cortisol release and schizotypy. Psychoneuroendocrinology 89: 209-215

Yung, Alison R, Lisa J Phillips, Patrick D McGorry, Colleen A McFarlane, Shona Francey, Susan Harrigan, George C Patton, and Henry J Jackson. 1998. 'Prediction of psychosis: a step towards indicated prevention of schizophrenia', The British Journal of Psychiatry

Yung AR, Yung AR, Yuen HP, Mcgorry PD, Phillips LJ, Kelly D, Dell'olio M, Francey SM, Cosgrave EM, Killackey E (2005) Mapping the onset of psychosis: the comprehensive assessment of at-risk mental states. Australian \& New Zealand Journal of Psychiatry 39:964-971

Zhang XY, Zhou DF, Cao LY, Wu GY, Shen YC (2005) Cortisol and cytokines in chronic and treatment-resistant patients with schizophrenia: association with psychopathology and response to antipsychotics. Neuropsychopharmacology 30:1532

Zuardi AW, Shirakawa I, Finkelfarb E, Karniol IG (1982) Action of cannabidiol on the anxiety and other effects produced by $\Delta 9$ THC in normal subjects. Psychopharmacology 76:245-250

Zuardi AW, Cosme RA, Graeff FG, Guimarães FS (1993) Effects of ipsapirone and cannabidiol on human experimental anxiety. J Psychopharmacol 7:82-88

Zuardi, Antonio W, Natália P Rodrigues, Angélica L Silva, Sandra A Bernardo, Jaime EC Hallak, Francisco S Guimarães, and José AS Crippa. 2017. 'Inverted U-shaped dose-response curve of the anxiolytic effect of cannabidiol during public speaking in real life', Frontiers in pharmacology, 8

Publisher's note Springer Nature remains neutral with regard to jurisdictional claims in published maps and institutional affiliations. 